\title{
KEYNOTE-676: Phase III study of BCG and pembrolizumab for persistent/recurrent high-risk NMIBC
}

\author{
Ashish M Kamat*,1, Neal Shore², Noah Hahn³, Shaheen Alanee ${ }^{4}$, Hiroyuki Nishiyama ${ }^{5}$, \\ Shahrokh Shariat ${ }^{6}$, Kijoeng Nam ${ }^{7}$, Ekta Kapadia ${ }^{8}$, Tara Frenkl ${ }^{8}$ \& Gary Steinberg ${ }^{9}$ \\ ${ }^{1}$ Department of Urology, The University of Texas MD Anderson Cancer Center, Houston, TX 77030, USA \\ ${ }^{2}$ Department of Urology, Carolina Urologic Research Center, Myrtle Beach, SC 29572, USA \\ ${ }^{3}$ Department of Oncology \& Department of Urology, Johns Hopkins University School of Medicine, Baltimore, MD 21287, USA \\ ${ }^{4}$ Department of Urology, Henry Ford Hospital, Detroit, Ml 48202, USA \\ ${ }^{5}$ Department of Urology, University of Tsukuba, Tsukuba 305-8577, Japan \\ ${ }^{6}$ Department of Urology, Medical University of Vienna, 1090 Vienna, Austria \\ ${ }^{7}$ Department of Biostatistics and Research Decision Sciences, Merck \& Co., Inc., Kenilworth, NJ 07033, USA \\ ${ }^{8}$ Department of Oncology, Merck \& Co., Inc., Kenilworth, NJ 07033, USA \\ ${ }^{9}$ Department of Urology, NYU Langone Health, New York, NY 10017, USA \\ *Author for correspondence: Tel.: +1 713792 3250; Fax: +1 713794 4824; akamat@mdanderson.org
}

Background: Nonmuscle-invasive bladder cancer (NMIBC) is the most common form of bladder cancer, with high rates of disease recurrence and progression. Current treatment for high-risk NMIBC involves Bacillus Calmette-Guérin (BCG) therapy, but treatment options are limited for patients with recurrent or BCGunresponsive disease. Aberrant programmed death 1 signaling has been implicated in BCG resistance and bladder cancer recurrence and progression, and pembrolizumab has shown efficacy in patients with BCGunresponsive high-risk NMIBC. Aim: To describe the rationale and design for the randomized, comparatorcontrolled Phase III KEYNOTE-676 study, which will evaluate the efficacy and safety of pembrolizumab in combination with BCG in patients with persistent/recurrent high-risk NMIBC after BCG induction therapy.

Trial registration number: NCT03711032

First draft submitted: 11 December 2019; Accepted for publication: 13 February 2020; Published online: 12 March 2020

Keywords: Bacillus Calmette-Guérin • bladder cancer • immunotherapy • nonmuscle-invasive bladder cancer • pembrolizumab $\bullet$ programmed death $1 \bullet$ programmed death ligand 1

Bladder cancer is the sixth most common cancer in the USA, accounting for $4.6 \%$ of all new cancer diagnoses [1]. This equates to an estimated 80,470 new diagnoses and 17,670 deaths in the USA in 2019 [1]. The prevalence of bladder cancer increases with age and more commonly affects men; the incidence of new diagnoses among men and women is 35 and nine cases per 100,000 person-years, respectively [1].

Nonmuscle-invasive bladder cancer (NMIBC) is the most common type of bladder cancer, accounting for $75-80 \%$ of all initial diagnoses [2-4] and comprising noninvasive papillary carcinomas (pathologic stage Ta; $48 \%$ ), submucosal invasive tumors (T1;27\%) and carcinoma in situ (CIS; 2\%) $[2,5]$. Furthermore, NMIBC may be categorized as low-, intermediate- or high-risk, with high-risk disease defined as any CIS, T1 or high-grade Ta [5].

Standard treatment for high-risk NMIBC involves transurethral resection of a bladder tumor followed by intravesical Bacillus Calmette-Guérin (BCG) induction and maintenance therapy for up to 3 years $[5,6]$. BCG is a live-attenuated strain of Mycobacterium bovis that induces an immune response in the bladder when administered [7]. Although approximately $70 \%$ of patients with NMIBC achieve complete response (CR) with BCG therapy, many patients with high-risk disease do not maintain remission [8-13]. As many as $60 \%$ of patients can have disease recurrence within 1 year, increasing to $80 \%$ within 15 years $[9,13]$. In addition, within 5 years, up to approximately $20-40 \%$ of patients who experience BCG failure will also experience progression to muscle invasive disease [1417] and approximately $6-15 \%$ of patients will have metastasis to the lymph nodes [18-20]. Accordingly, patients with high-risk NMIBC have ongoing, even life-long, monitoring and therapy requirements, including regular 
cystoscopies and biopsies, resulting in a reduced quality of life (QoL) for patients and high ongoing monitoring costs [21-23].

Another treatment option for patients with recurrent high-risk NMIBC or BCG-unresponsive disease is intravesical chemotherapy; agents are gemcitabine, mitomycin and valrubicin [3,6,24]. Published results of studies do not report improvements in efficacy with intravesical gemcitabine or mitomycin relative to intravesical BCG in intermediate- to high-risk NMIBC [25-27], although intravesical valrubicin can be useful to select patients with CIS [28]. However, a recent meta-analysis to examine the use of intravesical chemotherapy in combination with BCG reported significant improvements in recurrence-free survival (RFS), overall survival (OS) and disease-specific survival (DSS), compared with BCG alone in patients with intermediate- to high-risk NMIBC [29]. Several studies have also been conducted to investigate combination therapy with BCG and mitomycin C or interferon to improve efficacy and reduce disease recurrence and progression, although results have been mixed and largely failed to demonstrate a clear benefit over BCG monotherapy [30,31]. If intravesical treatment options fail, radical cystectomy with intestinal diversion is the recommended course $[3,6,24,32,33]$. Radical cystectomy may prevent local progression but also carries a high risk for postoperative complications, reduced QoL for patients and the potential for overtreatment of patients whose disease may not have progressed [33-35]. Additionally, not all patients are eligible for cystectomy because of advanced age or severe co-morbidities [6]. Because of the life-altering consequences and reduced QoL associated with radical cystectomy, many patients refuse to undergo the surgery [36]. Therefore, there remains an urgent need for novel therapies to provide a nonsurgical second-line treatment option.

\section{KEYNOTE-676 trial}

Here, we describe the rationale and design for the randomized, comparator-controlled Phase III KEYNOTE-676 study (NCT03711032), which will evaluate the efficacy and safety of pembrolizumab plus BCG compared with BCG monotherapy in patients with persistent/recurrent high-risk NMIBC after BCG induction therapy.

\section{Background \& rationale}

BCG is thought to exert its antitumor effects through direct and indirect mechanisms [12]. In addition to triggering apoptosis or necrosis upon internalization by bladder cancer cells (direct effect), BCG increases antigen expression and cytokine release from tumor cells, leading to immune cell (e.g., granulocytes, $\mathrm{CD} 4^{+}$and $\mathrm{CD} 8^{+}$lymphocytes, natural killer cells, macrophages) recruitment and immune-mediated cytotoxicity (indirect effect) [12].

PD-1 is an immune checkpoint receptor expressed on activated $\mathrm{T}$ cells that modulates immune response [37]. Tumor cells may hijack the interaction between PD-1 and its ligands PD-L1 and PD-L2 to avoid immunorecognition, for example, by expressing PD-L1, which binds PD-1 on activated T cells, allowing tumor cells to escape immune-mediated cytotoxicity [37,38]. Altered PD-1 pathway activation has been implicated in disease recurrence and progression and in BCG resistance in patients with NMIBC [39-43]. In particular, elevated PD-L1 expression has been observed across all tumor stages [39] and correlates with localized disease progression [40] and PD-1 expression on tumor-infiltrating lymphocytes [39]. Moreover, tumor PD-L1 expression is significantly increased after BCG treatment and is associated with reduced 5-year recurrence and OS (42 and $81 \%$ vs 55 and 100\% in patients with high and low tumor PD-L1 expression, respectively) [42,43]. Therefore, targeting the PD-1/PD-L1 pathway has emerged as a potential therapeutic option for patients with bladder cancer.

Pembrolizumab is a humanized monoclonal antibody that binds PD-1 with high affinity and selectivity, preventing PD-1 from interacting with its ligands PD-L1 and PD-L2 [44]. Pembrolizumab provides effective and durable antitumor activity against multiple cancers and has been approved for use in more than 60 countries [37,44]. The effectiveness of pembrolizumab monotherapy in treating locally advanced and metastatic urothelial carcinoma has previously been demonstrated in the Phase III KEYNOTE-0 45 (NCT02256436) [45] and Phase II KEYNOTE-052 (NCT02335424) studies [46].

The KEYNOTE-045 study reported significant survival benefits for second-line pembrolizumab in 542 patients with advanced urothelial cancer that had recurred or progressed after platinum-based chemotherapy [45]. After a median follow-up of 27.7 months, pembrolizumab resulted in an ongoing response with significantly longer OS (10.1 vs 7.3 months; $\mathrm{p}<0.001)$ and a higher objective response rate $(21.1$ vs $11.0 \%)$ than investigator-choice chemotherapy [47]. The relative benefit of pembrolizumab was observed across all levels of PD-L1 expression [47].

First-line pembrolizumab also demonstrated durable antitumor activity in 370 chemotherapy-naive cisplatinineligible patients with locally advanced unresectable or metastatic urothelial cancer who were enrolled in KEYNOTE-052 [46]. Tumor PD-L1 combined positive score (CPS) $\geq 10$ was associated with a higher frequency 
of response to pembrolizumab treatment ( $47.3 \%$ objective response rate vs $20.3 \%$ with PD-L1 CPS $<10)$, longer median OS (18.5 vs 9.8 months) and more durable response (median duration of response [DOR] was not reached for CPS $\geq 10$ and was 18.2 months for CPS $<10)[46,48]$.

Based on the promising antitumor activity of pembrolizumab demonstrated in the KEYNOTE-045 and KEYNOTE-052 studies in patients with advanced bladder cancer, an additional clinical study was initiated to evaluate pembrolizumab monotherapy in patients with NMIBC. KEYNOTE-057 (NCT02625961) is an openlabel Phase II study of pembrolizumab monotherapy (200 mg every 3 weeks for up to 2 years) in patients with BCG-unresponsive, high-risk NMIBC, divided into two cohorts: patients with CIS with or without papillary disease (cohort A) and patients with papillary disease without CIS (cohort B). In an interim analysis of 102 patients enrolled in cohort A of the KEYNOTE-057 study, pembrolizumab demonstrated encouraging antitumor activity and durable response [49]. No patient with recurrent disease had experienced progression to muscle-invasive or metastatic disease while on study therapy, based on protocol-specified disease assessments [49]. Recently, pembrolizumab monotherapy was approved by the US FDA for patients with BCG-unresponsive CIS who were ineligible for or declined to undergo cystectomy [44]. Additionally, a preliminary analysis of a Phase I study of intravesical BCG combined with pembrolizumab in patients with high-grade NMIBC after treatment failure with at least two courses on intravesical therapy (one contains BCG) showed that the combination therapy had an overall response rate of $67 \%$ and was well tolerated with acceptable safety [50].

Pembrolizumab has also offered a favorable safety profile compared with systemic chemotherapy, with a lower incidence of adverse events (AEs), including serious AEs [47]. Furthermore, the proportion of patients administered pembrolizumab who discontinue treatment is relatively low compared with chemotherapy, and the lower risk for cumulative toxicity with pembrolizumab may facilitate a longer duration of treatment [47]. In KEYNOTE-057, the safety profile of pembrolizumab monotherapy was consistent with the previously established safety profile of pembrolizumab in advanced urothelial and other cancers [44]. Most AEs reported in this study were grade 1 or 2 and were effectively managed [49]. The possible role of immune checkpoint signaling in BCG resistance and the efficacy of pembrolizumab in patients with BCG-unresponsive high-risk NMIBC suggest that combining immune checkpoint blockade and BCG therapy may increase the antitumor activity of BCG-based therapy.

\section{Study design}

The KEYNOTE-676 study is a randomized, comparator-controlled, open-label Phase III study of BCG with or without pembrolizumab in patients with high-risk NMIBC that is persistent or recurrent after adequate BCG induction therapy (Figure 1). Patients who received BCG maintenance or a second induction are not eligible. Eligible patients are randomly assigned in a 1:1 ratio to receive pembrolizumab $200 \mathrm{mg}$ administered by intravenous infusion every 3 weeks plus BCG $50 \mathrm{mg}$ induction therapy administered via intravesical instillation (once weekly for 6 weeks) or BCG monotherapy. BCG induction therapy is followed by maintenance therapy (once weekly for 3 weeks at months 3, 6, 12, 18, 24, 30 and 36) in both arms. Treatment will continue until disease progression, persistent/recurrent high-risk disease, unacceptable toxicity, investigator or patient decision to withdraw, pregnancy, nonadherence to treatment or trial procedures, or completion of 35 cycles of pembrolizumab ( $\sim 2$ years) or 145 weeks of treatment with BCG $(\sim 3$ years).

Randomization will be conducted using an interactive response technology system, and patients will be stratified according to histology (CIS vs non-CIS), PD-L1 status (CPS $\geq 10$ vs CPS $<10$ ) and NMIBC disease history (persistent/recurrent NMIBC at $0-\leq 6$ months vs recurrent NMIBC at $>6-\leq 12$ months vs $>12-\leq 24$ months). Because this is an open-label study, both patients and investigators will know treatment assignment.

The study protocol and all amendments were approved by the institutional review board or independent ethics committee of each participating institution. The trial was conducted in accordance with the provision of the Declaration of Helsinki, Good Clinical Practice guidelines, and all local laws and regulations. All patients provided written informed consent.

\section{Eligibility criteria}

Eligibility criteria are described in Table 1 . Briefly, men and women aged $\geq 18$ years are eligible for enrollment if they have a histologically confirmed diagnosis of high-risk nonmuscle invasive (i.e., high-grade papillary carcinoma [Ta], submucosal invasive tumor [T1] and/or CIS) transitional cell carcinoma that is persistent (Ta and/or CIS) or recurrent (Ta, T1 and/or CIS) after one adequate course of BCG induction therapy. 


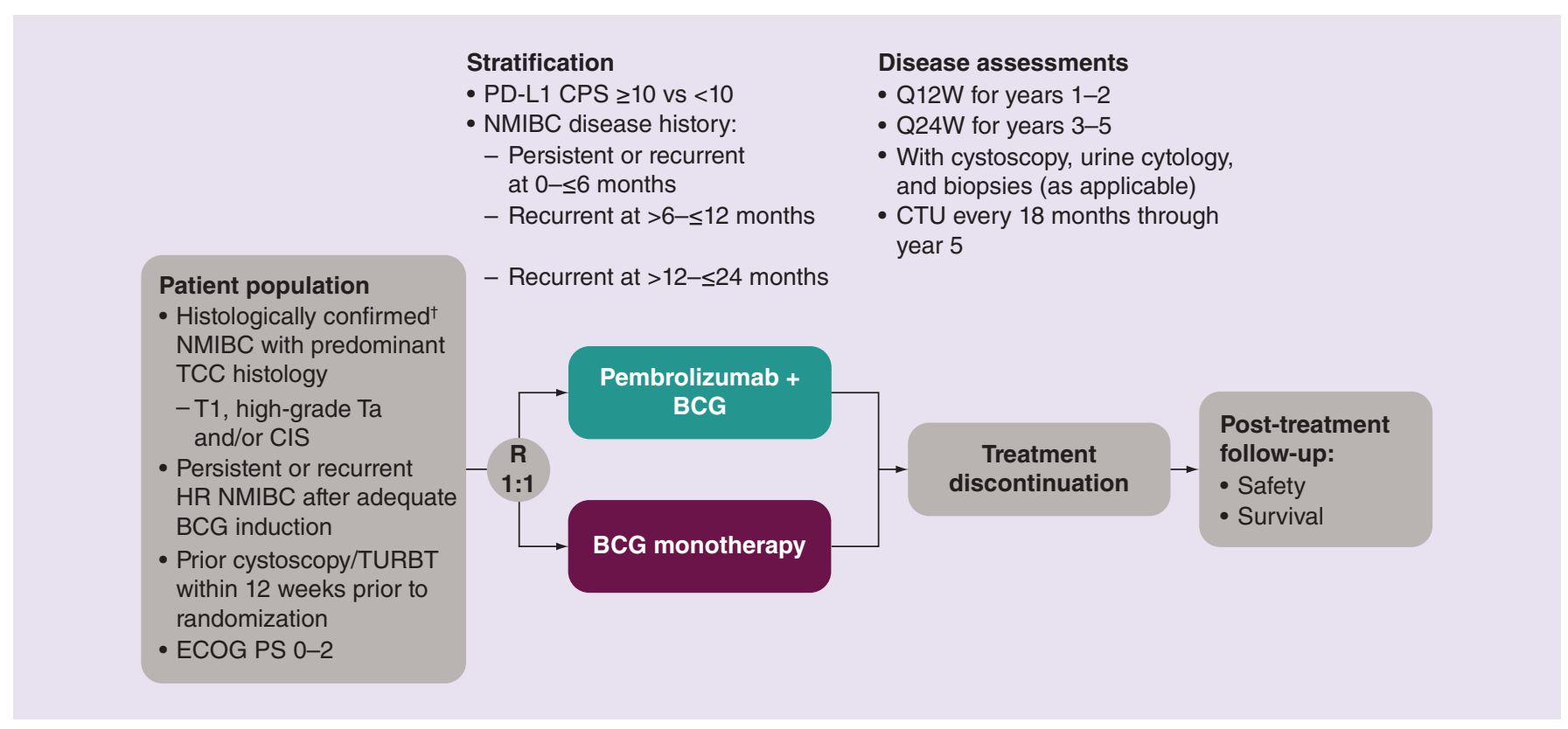

Figure 1. KEYNOTE-676 study design.

${ }^{\dagger}$ Assessed by blinded independent central review.

BCG: Bacillus Calmette-Guérin; CIS: Carcinoma in situ; CPS: Combined positive score; CTU: Computed tomography urography; ECOG PS:

Eastern Cooperative Oncology Group performance status; HR: High-risk; NMIBC: Nonmuscle-invasive bladder cancer; PD-L1: Programmed death ligand 1; Q12W: Every 12 weeks; Q24W: Every 24 weeks; R: Randomized; T1: Submucosal invasive tumor; Ta: Noninvasive papillary carcinoma; TCC: Transitional cell carcinoma; TURBT: Transurethral resection of bladder tumor.

\section{Planned sample size \& study period}

The planned sample size is approximately 550 participants, including no more than 182 patients with non-CIS disease. KEYNOTE-676 started in December 2018, and the estimated study completion date is 25 November 2024 .

\section{Outcome measures/end points}

The primary end point is CR rate in patients with CIS. CR rate is defined as the absence of high-risk NMIBC as determined by blinded independent central review of urine cytology, biopsy and radiology assessments and local cystoscopy evaluation.

Secondary end points are event-free survival (EFS), 12-month EFS rate, RFS, OS, DSS, time to cystectomy, DOR, 12-month DOR rate, health-related QoL (assessed using the EORTC QoL Questionnaire Core 30 [QLQC30], the EORTC NMIBC-specific questionnaire [QLQ-NMIBC24] and the EuroQol 5-dimension, 5-level questionnaire [EQ-5D-5L]) and safety and tolerability. Exploratory end points are identification of biomarkers that may be indicative of clinical response or resistance, safety, pharmacodynamic activity and the mechanism of action of pembrolizumab.

\section{Study procedures}

Disease will be assessed using integrated cystoscopy and blinded independent central review of urine cytology and biopsy as applicable (Figure 2). Based on investigator discretion, cystoscopy will be performed via white light cystoscopy or fluorescence-guided cystoscopy (photodynamic diagnosis or blue light cystoscopy) to inspect the entire urothelial lining.

Initial disease assessment will be performed during screening (within 12 weeks of randomization) followed by every 12 weeks for years $1-2$, then every 24 weeks for years $3-5$. Computed tomography urography will be conducted at screening, 18 months after randomization and then every 18 months for patients who have not had a high-grade recurrence, or more frequently, if clinically indicated. Patients will be followed-up for survival status by phone, e-mail or other means approximately every 12 weeks until death, withdrawal of consent or the end of the study. 
Table 1. Eligibility criteria for KEYNOTE-676.

\section{Inclusion criteria}

\section{- Age $\geq 18$ years}

- Histologically confirmed diagnosis of NMIBC

(high-grade Ta, T1 and/or CIS) with

TCC-predominant histology

- Completed 1 adequate course of BCG induction

therapy for the treatment of high-risk NMIBC

- Persistent ${ }^{\dagger}$ or recurrent ${ }^{\ddagger}$ high-risk NMIBC after

- Underwent TURBT to remove all resectable

disease within 12 weeks before randomization to

confirm absence of muscle invasion

- Provide tissue for biomarker analysis from most recent TURBT (or archival tissue if recent tissue is insufficient)

- ECOG PS 0,1 or 2

- Adequate organ function study and for 120 days after the last dose of study treatment adequate induction therapy

- Willing to use contraception throughout the

\section{Exclusion criteria}

\section{- Predominant or exclusive non-TCC}

- $>1$ induction course of BCG, or any maintenance therapy of BCG

- Persistent ${ }^{\dagger} \mathrm{T} 1$ disease after BCG induction

- Muscle invasive (T2, T3, T4), locally advanced nonresectable or metastatic urothelial carcinoma

- Concurrent extravesical (urethra, ureter, renal pelvis) NMIBC TCC, concurrent upper tract

involvement, invasive prostatic TCC or ductal invasion

- Previous therapy with an anti-PD-1, anti-PD-L1 or anti-PD-L2 agent or agent directed to another stimulatory or coinhibitory T-cell receptor (e.g., CTLA-4, OX-40, CD137)

- Previous systemic anticancer therapy $\leq 4$ weeks from start of treatment or prior radiotherapy

$\leq 2$ weeks from start of treatment

- Known additional malignancy that is progressing or has required active treatment in the past 3 years (except for patients with BCC or SCC of the skin that underwent potentially curative treatment; or a history of prostate cancer $\left[\leq \mathrm{T}_{2} \mathrm{~N}_{0} \mathrm{M}_{0}\right]$ treated with surgery or radiotherapy $\geq 1$ year before study entry and acceptable PSA levels)

- Diagnosis of immunodeficiency or long-term systemic steroid therapy or immunosuppressive

therapy within 7 days before first dose of study treatment

- Active autoimmune disease necessitating systemic treatment in the preceding 2 years

- Active infection necessitating systemic therapy

- Received a live vaccine within 30 days from start of treatment

- Current pneumonitis or history of noninfectious pneumonitis that necessitated steroid therapy

- Known hypersensitivity to pembrolizumab

- $\geq 1$ contraindication to BCG (e.g., prior BCG sepsis or systemic infection, total bladder incontinence, adverse experience to previous BCG instillation that resulted in treatment discontinuation and precludes treatment)

- Pregnant or breastfeeding, or expecting to conceive within projected study duration

- Known history of HIV, hepatitis B or hepatitis C infection

- Known psychiatric or substance abuse disorder that would interfere with cooperation with study requirements

- Participation in a study of an investigational agent or device within 4 weeks of start of study treatment

${ }^{\dagger}$ NMIBC present within 3-6 months after starting BCG induction therapy. Patients with persistent T1 disease are not eligible for the study.

$\ddagger$ Reappearance of high-risk NMIBC after CR or tumor-free state within 6 months of starting BCG induction. Recurrence must be within 24 months of last exposure to BCG.

BCC: Basal cell carcinoma; BCG: Bacillus Calmette-Guérin; CIS: Carcinoma in situ; CR: Complete response; ECOG PS: Eastern Cooperative Oncology Group performance status; NMIBC: Nonmuscle-invasive bladder cancer; SCC: Squamous cell carcinoma; T1: Submucosal invasive tumor; Ta: Noninvasive papillary carcinoma; TCC: Transitional cell carcinoma; TURBT: Transurethral resection of bladder tumor.

Safety will be monitored throughout the study and for 30 days at the end of study treatment ( 90 days for serious AEs). Safety assessments are incidence, causality and outcome of AEs and changes in vital signs and laboratory values. AEs will be graded and recorded throughout the study and follow-up period using the Common Terminology Criteria for Adverse Events, version 4.0.

Patient-reported outcome (PRO) assessments (QLQ-C30, QLQ-NMIBC24, EQ-5D-5L) will be administered electronically at baseline and at regular intervals during the study. PD-L1 status will be determined by immunohistochemistry at screening. The exploratory biomarker investigation may include blood and tumor DNA, RNA and proteomic investigations and urine cytokine analysis.

\section{Statistics}

Efficacy (EFS, RFS, OS, DSS and time to cystectomy) end points will be assessed in the intention-to-treat population and analyzed by randomized treatment group. The primary hypothesis for CR rate will be evaluated in the CIS subgroup of the intention-to-treat population by comparing pembrolizumab plus BCG with BCG monotherapy using the stratified Miettinen and Nurminen method [51]. Similarly, DOR will be analyzed for responders with documented CR in the subset of patients with CIS. EFS, OS, RFS, DSS and time to cystectomy will be evaluated using a stratified Cox proportional hazards model. Twelve-month EFS rate and 12-month DOR rates will be estimated using the Kaplan-Meier method.

Safety will be assessed in all randomly assigned patients who received study treatment according to study treatment received and will be analyzed using a tiered approach. PROs will be assessed in the PRO full analysis set population, comprising patients who have received treatment and have $\geq 1$ PRO assessment available.

Interim analyses are planned. The external data monitoring committee will routinely review safety and efficacy data and make recommendations for study discontinuation and modifications accordingly. 


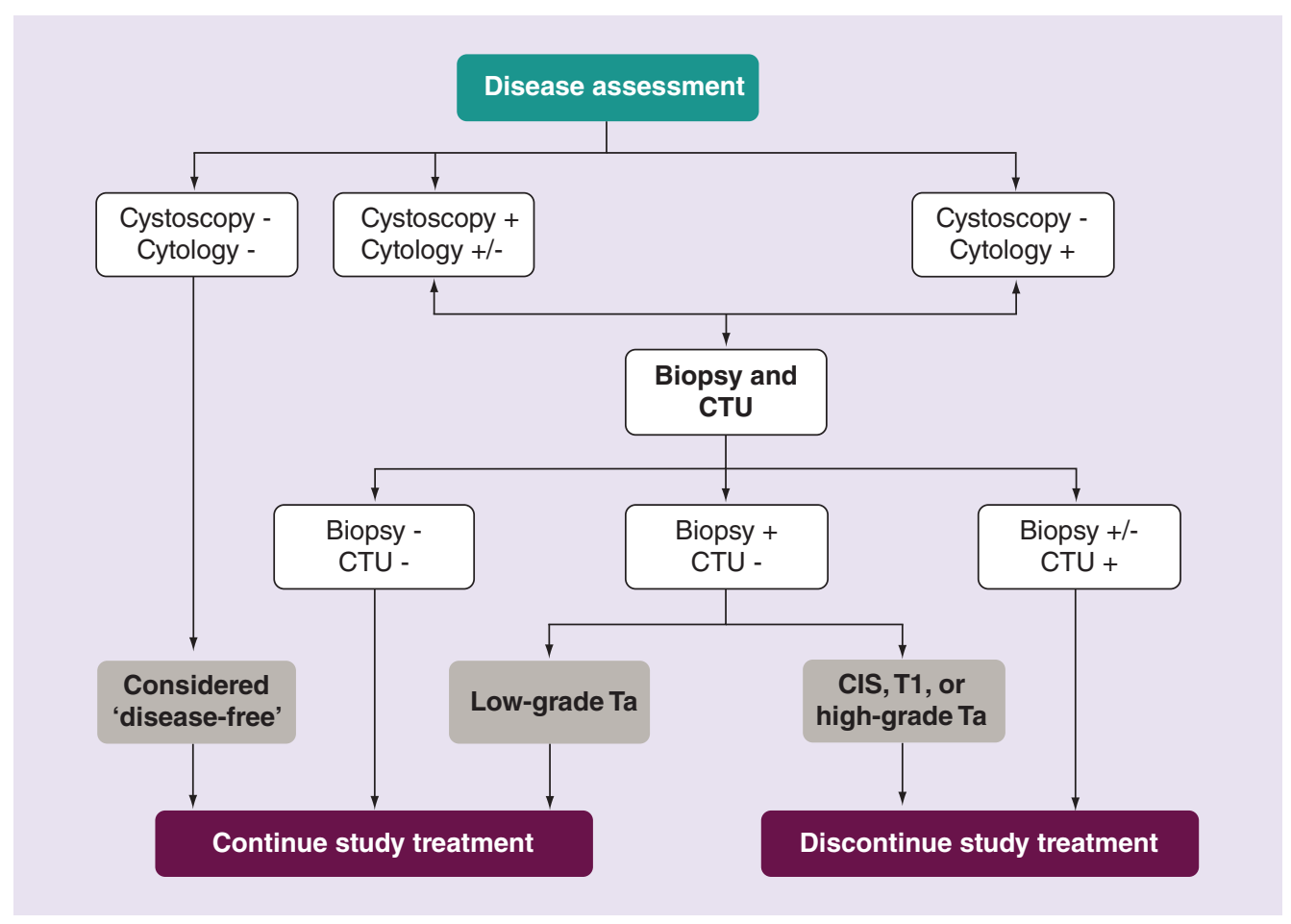

Figure 2. Disease assessment decision tree.

CTU +/-: Positive/negative for extravesical disease showing upper urinary tract or visceral/nodal disease consistent with bladder cancer.

Cytology+: Positive for or suggestive of malignant cells (central vendor interpretation: 'suggestive of high-grade urothelial carcinoma' or 'high-grade urothelial carcinoma').

Cytology-: Not indicative or suggestive of malignant cells (central vendor interpretation: 'negative for high-grade urothelial carcinoma', 'atypical urothelial cells present' or 'low-grade urothelial neoplasm').

Cystoscopy +/-: Indicative or suggestive of malignancy/without malignant or suspicious lesions.

CIS: Carcinoma in situ; CTU: Computed tomography urography; T1: Submucosal invasive tumor; Ta: Noninvasive papillary carcinoma.

\section{Conclusion}

Aberrant immune checkpoint pathway activation has been implicated in NMIBC disease recurrence and progression and may also mediate BCG resistance mechanisms. Preliminary data from the KEYNOTE-057 study indicate that pembrolizumab may be effective in patients with BCG-unresponsive high-risk NMIBC. These data support the hypothesis that greater antitumor activity in high-risk NMIBC can be achieved by combining immune checkpoint inhibition and BCG therapy. Here, we described the methodology for the KEYNOTE-676 study, an ongoing open-label, comparator-controlled Phase III study to evaluate the efficacy and safety of pembrolizumab-BCG combination therapy for patients with persistent/recurrent high-risk NMIBC after BCG induction. The results from the KEYNOTE-676 study may indicate a more effective treatment option for patients at high risk for recurrent or progressive disease. 
Summary points

\section{Background}

- Effective treatment options are limited for patients with recurrent or persistent high-risk nonmuscle-invasive bladder cancer (NMIBC), or Bacillus Calmette-Guérin (BCG)-unresponsive disease.

Rationale

- Preliminary results from the Phase II KEYNOTE-057 study in patients with BCG-unresponsive high-risk NMIBC indicate that pembrolizumab provides effective antitumor activity and an acceptable safety profile.

- Pembrolizumab has also demonstrated efficacy and safety in patients with advanced or metastatic bladder cancer in the KEYNOTE-045 and KEYNOTE-052 studies.

- Combining pembrolizumab and BCG therapies may provide anticancer activity superior to that of BCG monotherapy for patients with recurrent or persistent high-risk NMIBC.

KEYNOTE-676 study design \& eligibility criteria

- KEYNOTE-676 is a randomized, open-label, comparator-controlled Phase III study designed to evaluate the efficacy and safety of pembrolizumab plus BCG compared with BCG monotherapy for patients with persistent or recurrent high-risk NMIBC after BCG induction therapy.

- Approximately 550 patients with high-grade papillary carcinoma (Ta), submucosal invasive tumor (T1) and/or carcinoma in situ, that is persistent or recurrent after one adequate course of BCG induction therapy will be enrolled.

- Eligible patients will be randomly assigned to receive pembrolizumab plus BCG or BCG alone.

Outcome measures/end points

- The primary end point is complete response rate in patients with carcinoma in situ; secondary end points are event-free survival, 12-month event-free survival rate, recurrence-free survival, overall survival, disease-specific survival, time to cystectomy, duration of response, 12-month duration of response rate, health-related quality of life and safety and tolerability.

Conclusion

- Results from the KEYNOTE-676 trial may indicate a more effective treatment option for patients with high-risk NMIBC.

Future perspective

- In 5-10 years, inhibition of the programmed death 1 /ligand 1 pathway will become a significant treatment option for patients with NMIBC in order to delay radical cystectomy.

\section{Author contributions}

AM Kamat, N Hahn, S Shariat, S Alanee, T Frenkl, E Kapadia and G Steinberg contributed to the conception, design or planning of the study. AM Kamat, S Shariat, N Shore, K Nam, E Kapadia and G Steinberg contributed to the analysis of the data. AM Kamat, H Nishiyama, K Nam and E Kapadia contributed to the acquisition of the data. AM Kamat, N Shore, H Nishiyama, K Nam, E Kapadia and $G$ Steinberg contributed to the interpretation of the results. AM Kamat, N Shore and T Frenkl contributed to the drafting of the manuscript. AM Kamat contributed to administrative, logistical or technical support. All the authors reviewed or revised the manuscript for important intellectual content and approved the final draft for submission.

\section{Acknowledgments}

The authors thank the patients and their families and all investigators and site personnel. The authors also thank Kentaro Imai (employee of Merck Sharp \& Dohme, a subsidiary of Merck \& Co., Inc., Kenilworth, NJ, USA) for his contributions to the development of the manuscript.

Financial \& competing interests disclosure

Funding for this research was provided by Merck Sharp \& Dohme Corp., a subsidiary of Merck \& Co., Inc., NJ, USA. AM Kamat has received honoraria from UroToday Publishing, EAU (EU Oncology); has a consultant/advisory role for Merck, Bristol-Myers Squibb, Imain, Eisai, Arquer, MDX Health, Photocure, AstraZeneca, Tesaro, Abbott Molecular, US Biotest and Ferring; has received research funding from FKD Industries and Merck; has patents/royalties for CyPRIT (Cytokine Panel for Response to Intravesical Immunotherapy); has received travel and accommodations expenses from Pfizer Japan and is the president of the International Bladder Cancer Group, International Bladder Cancer Network. N Shore has a consultant/advisory role for Amgen, Astellas, AstraZeneca, Bayer, Bristol-Myers Squibb, Dendreon, Ferring, Janssen, Merck, MDxHealth, Pfizer, Sanofi and Tolmar and is a member of a speakers' bureau for Astellas and Janssen. $\mathrm{N} \mathrm{Hahn} \mathrm{is} \mathrm{a} \mathrm{consultant} \mathrm{for} \mathrm{Bristol-Myers} \mathrm{Squibb,} \mathrm{Champions} \mathrm{Oncology,}$ CicloMed, Ferring, Genentech, Health Advances, Incyte, Inovio Janssen, Merck, Principia Biopharma, Rexahn, Seattle Genetics, TARDIS Biomedical, Astex, AstraZeneca and Pieris and has received honoraria from the Bladder Cancer Academy and PierView. 
S Alanne has a consultant/advisory role with Merck and AstraZeneca, has received research funding from Merck, and has received travel/accommodations/expenses from AstraZeneca. $\mathrm{H}$ Nishiyama received personal fees during the conduct of the study from Merck Sharp \& Dohme. S Shariat has received honoraria and travel expenses/accommodations from Astellas, AstraZeneca, Bayer, Bristol-Myers Squibb, Cepheid, Ferring, Ipsen, Janssen, Lilly, Merck, Olympus, Pfizer, Pierre Fabre, Richard Wolf, Roche, Sanochemia, Sanofi, Takeda and Urogen; is a stockholder for Urogen and Sanochemia; and has received research funding (institution) from Sanofi, Roche and Astellas. K Nam and E Kapadia are employees of Merck Sharp \& Dohme, a subsidiary of Merck \& Co., Inc., Kenilworth, NJ, USA and stockholders in the company. T Frenkl was an employee of Merck Sharp \& Dohme, a subsidiary of Merck \& Co., Inc., Kenilworth, NJ, USA, at the time of development of this manuscript and a stockholder in the company and is a current employee of GlaxoSmithKline. G Steinberg had a consultant/advisory role for Merck, Cold Genesys, Urogen, Fidia, Ferring, FKD, MDxHealth, Photocure, Natera, QED Therapeutics and Taris Biomedical. The authors have no other relevant affiliations or financial involvement with any organization or entity with a financial interest in or financial conflict with the subject matter or materials discussed in the manuscript apart from those disclosed.

Medical writing and/or editorial assistance was provided by Matthew Grzywacz, PhD, of the ApotheCom pembrolizumab team (Yardley, PA, USA). This assistance was funded by Merck Sharp \& Dohme Corp., a subsidiary of Merck \& Co., Inc., Kenilworth, NJ, USA.

\section{Open access}

This work is licensed under the Attribution-NonCommercial-NoDerivatives 4.0 Unported License. To view a copy of this license, visit http://creativecommons.org/licenses/by-nc-nd/4.0/

\section{References}

Papers of special note have been highlighted as: $\bullet$ of interest; $\bullet \bullet$ of considerable interest

1 National Cancer Institute. SEER cancer stat facts: bladder cancer (2019). https://seer.cancer.gov/statfacts/html/urinb.html

2 Boustead GB, Fowler S, Swamy R, Kocklebergh R, Hounsome L. Stage, grade and pathological characteristics of bladder cancer in the UK: British Association of Urological Surgeons (BAUS) urological tumour registry. BJU Int. 113(6), 924-930 (2014).

3 Babjuk M, Bohle A, Burger M et al. EAU guidelines on non-muscle-invasive urothelial carcinoma of the bladder: update 2016. Eur. Urol. 71(3), 447-461 (2017).

4 NICE Guidance. Bladder cancer: diagnosis and management of bladder cancer. BJU Int. 120(6), 755-765 (2017).

5 Chang SS, Boorjian SA, Chou R et al. Diagnosis and treatment of non-muscle invasive bladder cancer: AUA/SUO guideline. J. Urol. 196(4), 1021-1029 (2016).

6 National Comprehensive Cancer Network. NCCN clinical practice guidelines in oncology. Bladder Cancer (2019). www.nccn.org/professionals/physician_gls/pdf/bladder.pdf

7 BCG live (for intravesical use) TICE® BCG [package insert]. Organon USA, NJ, USA (2009). www.fda.gov/media/76396/download

8 Millan-Rodriguez F, Chechile-Toniolo G, Salvador-Bayarri J, Palou J, Algaba F, Vicente-Rodriguez J. Primary superficial bladder cancer risk groups according to progression, mortality and recurrence. J. Urol. 164(3 Pt 1), 680-684 (2000).

9 Sylvester RJ, Van Der Meijden AP, Oosterlinck W et al. Predicting recurrence and progression in individual patients with stage Ta T1 bladder cancer using EORTC risk tables: a combined analysis of 2596 patients from seven EORTC trials. Eur. Urol. 49(3), 466-465, discussion 475-467 (2006).

10 Hemdan T, Johansson R, Jahnson S et al. 5-Year outcome of a randomized prospective study comparing bacillus Calmette-Guerin with epirubicin and interferon-alpha2b in patients with T1 bladder cancer. J. Urol. 191(5), 1244-1249 (2014).

11 Herr HW, Milan TN, Dalbagni G. BCG-refractory vs. BCG-relapsing non-muscle-invasive bladder cancer: a prospective cohort outcomes study. Urol. Oncol. 33(3), 108, e101-e104 (2015).

12 Saluja M, Gilling P. Intravesical bacillus Calmette-Guerin instillation in non-muscle-invasive bladder cancer: a review. Int. J. Urol. 25(1), 18-24 (2018).

13 Wang H, Ding W, Jiang G et al. EORTC risk tables are more suitable for Chinese patients with nonmuscle-invasive bladder cancer than AUA risk stratification. Medicine 97(36), e12006 (2018).

14 Kamat AM, Hahn NM, Efstathiou JA et al. Bladder cancer. Lancet 388(10061), 2796-2810 (2016).

15 Van Den Bosch S, Alfred Witjes J. Long-term cancer-specific survival in patients with high-risk, non-muscle-invasive bladder cancer and tumour progression: a systematic review. Eur. Urol. 60(3), 493-500 (2011).

16 Chang SS, Boorjian SA, Chou R et al. Diagnosis and treatment of non-muscle invasive bladder cancer: AUA/SUO guideline. J. Urol. 196(4), 1021-1029 (2016).

17 Hussain MHA, Wood DP, Bajorin DF et al. Bladder cancer: narrowing the gap between evidence and practice. J. Clin. Oncol. 27(34), 5680-5684 (2009). 
18 Wiesner C, Pfitzenmaier J, Faldum A. Lymph node metastases in non-muscle invasive bladder cancer are correlated with the number of transurethral resections and tumor upstaging at radical cystectomy. Urol. Oncol. 95, 301-305 (2004).

19 Tilki D, Reich O, Svatek RS et al. Characteristics and outcomes of patients with clinical carcinoma in situ only treated with radical cystectomy: an international study of 243 patients. J. Urol. 183(5), 1757-1763 (2010).

20 Bruins HM, Skinner EC, Dorin RP et al. Incidence and location of lymph node metastases in patients undergoing radical cystectomy for clinical non-muscle invasive bladder cancer: results from a prospective lymph node mapping study. Urol. Oncol. 32(1), 24.e13-29.e13 (2014).

21 Mossanen M, Wang Y, Szymaniak J et al. Evaluating the cost of surveillance for non-muscle-invasive bladder cancer: an analysis based on risk categories. World J. Urol. 37(10), 2059-2065 (2019).

22 Heijnsdijk EaM, Nieboer D, Garg T, Lansdorp-Vogelaar I, De Koning HJ, Nielsen ME. Cost-effectiveness of surveillance schedules in older adults with non-muscle-invasive bladder cancer. BJU Int. 123(2), 307-312 (2019).

23 Jung A, Nielsen ME, Crandell JL et al. Quality of life in non-muscle-invasive bladder cancer survivors: a systematic review. Cancer Nurs. 42(3), E21-E33 (2019).

24 Gual Frau J, Palou J, Rodriguez O, Parada R, Breda A, Villavicencio H. Failure of Bacillus Calmette-Guerin therapy in non-muscle-invasive bladder cancer: definition and treatment options. Arch. Esp. Urol. 69(7), 423-433 (2016).

25 Schmidt S, Kunath F, Coles B et al. Intravesical Bacillus Calmette-Guérin versus mitomycin C for Ta and T1 bladder cancer. Cochrane Database Syst. Rev. 1, CD011935 (2020).

26 Porena M, Del Zingaro M, Lazzeri $\mathrm{M}$ et al. Bacillus Calmette-Guerin versus gemcitabine for intravesical therapy in high-risk superficial bladder cancer: a randomised prospective study. Urol. Int. 84(1), 23-27 (2010).

27 Prasanna T, Craft P, Balasingam G, Haxhimolla H, Pranavan G. Intravesical gemcitabine versus intravesical Bacillus Calmette-Guerin for the treatment of non-muscle invasive bladder cancer: an evaluation of efficacy and toxicity. Front. Oncol. 7, 260 (2017).

28 Cookson MS, Chang SS, Lihou C et al. Use of intravesical valrubicin in clinical practice for treatment of nonmuscle-invasive bladder cancer, including carcinoma in situ of the bladder. Ther. Adv. Urol. 6(5), 181-191 (2014).

29 Huang D, Jin YH, Weng H, Huang Q, Zeng XT, Wang XH. Combination of intravesical Bacille Calmette-Guerin and chemotherapy vs. Bacille Calmette-Guerin alone in non-muscle invasive bladder cancer: a meta-analysis. Front. Oncol. 9, 121 (2019).

30 Deng T, Liu B, Duan X, Zhang T, Cai C, Zeng G. Systematic review and cumulative analysis of the combination of mitomycin C plus Bacillus Calmette-Guerin (BCG) for non-muscle-invasive bladder cancer. Sci. Rep. 7(1), 3172 (2017).

31 Shepherd AR, Shepherd E, Brook NR. Intravesical Bacillus Calmette-Guerin with interferon-alpha versus intravesical Bacillus Calmette-Guerin for treating non-muscle-invasive bladder cancer. Cochrane Database Syst. Rev. 3, CD012112 (2017).

32 Shen PL, Lin ME, Hong YK, He XJ. Bladder preservation approach versus radical cystectomy for high-grade non-muscle-invasive bladder cancer: a meta-analysis of cohort studies. World J. Surg. Oncol. 16(1), 197 (2018).

33 Pang KH, Noon AP. Selection of patients and benefit of immediate radical cystectomy for non-muscle invasive bladder cancer. Transl. Androl. Urol. 8(1), 101-107 (2019).

34 Mason SJ, Downing A, Wright P et al. Health-related quality of life after treatment for bladder cancer in England. Br. J. Cancer 118(11), 1518-1528 (2018).

35 Winters BR, Wright JL, Holt SK, Dash A, Gore JL, Schade GR. Health related quality of life following radical cystectomy: comparative analysis from the medicare health outcomes survey. J. Urol. 199(3), 669-675 (2018).

36 Herr HW. Outcome of patients who refuse cystectomy after receiving neoadjuvant chemotherapy for muscle-invasive bladder cancer. Eur. Urol. 54(1), 126-132 (2008).

37 Stenehjem DD, Tran D, Nkrumah MA, Gupta S. PD1/PDL1 inhibitors for the treatment of advanced urothelial bladder cancer. OncoTargets Ther. 11, 5973-5989 (2018).

38 Iwai Y, Ishida M, Tanaka Y, Okazaki T, Honjo T, Minato N. Involvement of PD-L1 on tumor cells in the escape from host immune system and tumor immunotherapy by PD-L1 blockade. Proc. Natl Acad. Sci. USA 99(19), 12293-12297 (2002).

39 Boorjian SA, Sheinin Y, Crispen PL et al. T-cell coregulatory molecule expression in urothelial cell carcinoma: clinicopathologic correlations and association with survival. Clin. Cancer Res. 14(15), 4800-4808 (2008).

40 Inman BA, Sebo TJ, Frigola X et al. PD-L1 (B7-H1) expression by urothelial carcinoma of the bladder and BCG-induced granulomata: associations with localized stage progression. Cancer 109(8), 1499-1505 (2007).

41 Fukumoto K, Kikuchi E, Mikami S et al. Clinical role of programmed cell death-1 expression in patients with non-muscle-invasive bladder cancer recurring after initial Bacillus Calmette-Guerin therapy. Ann. Surg. Oncol. 25(8), 2484-2491 (2018).

-• Investigates programmed cell death ligand-1 (PD-L1) expression tumors before and after Bacillus Calmette-Guérin (BCG) treatment, and the association between PD-L1 expression in BCG-relapsing tumors and subsequent 5-year recurrence and progression.

42 Hashizume A, Umemoto S, Yokose T et al. Enhanced expression of PD-L1 in non-muscle-invasive bladder cancer after treatment with Bacillus Calmette-Guerin. Oncotarget 9(75), 34066-34078 (2018). 
-. Evaluation of PD-L1 and PD-L2 expression levels in nonmuscle-invasive bladder cancer tumor and nontumor tissues before and after patients received BCG therapy.

43 Nakanishi J, Wada Y, Matsumoto K, Azuma M, Kikuchi K, Ueda S. Overexpression of B7-H1 (PD-L1) significantly associates with tumor grade and postoperative prognosis in human urothelial cancers. Cancer Immunol. Immunother. 56(8), 1173-1182 (2007).

- Reports the association between PD-L1 expression and recurrence-free survival and overall survival in patients with urothelial cancer.

44 KEYTRUDA $^{\circledR}$ (Pembrolizumab) for injection, for intravenous use. Merck Sharp \& Dohme Corp., NJ, USA (2020). www.merck.com/product/usa/pi_circulars/k/keytruda/keytruda_pi.pdf

45 Bellmunt J, De Wit R, Vaughn DJ et al. Pembrolizumab as second-line therapy for advanced urothelial carcinoma. N. Engl. J. Med. 376(11), 1015-1026 (2017).

-. Multicenter, open-label Phase III study (KEYNOTE-045) in 542 patients to evaluate pembrolizumab as a second-line therapy for patients with advanced urothelial cancer that had recurred or progressed after platinum-based chemotherapy. Found superior efficacy of pembrolizumab compared with chemotherapy.

46 Balar AV, Castellano D, O’Donnell PH et al. First-line pembrolizumab in cisplatin-ineligible patients with locally advanced and unresectable or metastatic urothelial cancer (KEYNOTE-052): a multicentre, single-arm, Phase II study. Lancet Oncol. 18(11), 1483-1492 (2017).

-. Multicenter, single-arm, Phase II study (KEYNOTE-052) assessing objective response rates following pembrolizumab therapy in 370 chemotherapy-naive cisplatin-ineligible patients with locally advanced unresectable or metastatic urothelial cancer. Reported antitumor activity and an acceptable safety profile for pembrolizumab.

47 Fradet Y, Bellmunt J, Vaughn DJ et al. Randomized Phase III KEYNOTE-045 trial of pembrolizumab versus paclitaxel, docetaxel, or vinflunine in recurrent advanced urothelial cancer: results of $>2$ years of follow-up. Ann. Oncol. 30(6), 970-976 (2019).

48 Vuky J, Balar AV, Castellano DE et al. Updated efficacy and safety of KEYNOTE-052: a single-arm Phase II study investigating first-line pembrolizumab (pembro) in cisplatin-ineligible advanced urothelial cancer (UC). J. Clin. Oncol. 36, 4524 (2018).

49 De Wit R, Kulkarni GS, Uchio EM et al. Health-related quality of life and updated follow-up from KEYNOTE-057: Phase II study of pembrolizumab for patients with high-risk non-muscle invasive bladder cancer unresponsive to Bacillus Calmette-Guérin. Ann. Oncol. 30(Suppl. 5), v356-v402 (2019).

-• Results from the open-label Phase II KEYNOTE-057 study reporting safety and efficacy of pembrolizumab monotherapy for patients with BCG-unresponsive high-risk nonmuscle-invasive bladder cancer.

50 Alanee S, El-Zawahry A, McVary K et al. Phase I trial of intravesical Bacillus Calamette-Guérin combined with intravenous pembrolizuamb in high-grade nonmuscle invasive bladder cancer. Presented at: 20th Annual Meeting of the Society of Urologic Oncology. Washington DC, WA, USA, 4-6 December 2019.

51 Miettinen O, Nurminen M. Comparative analysis of two rates. Stat. Med. 4(2), 213-226 (1985). 